University of Wollongong

Research Online

Faculty of Informatics - Papers (Archive)

Faculty of Engineering and Information

Sciences

$1-1-2008$

\title{
Evaluation of human gait through observing body movements
}

Amir S. Hesami

University of Wollongong, ahesami@uow.edu.au

Fazel Naghdy

University of Wollongong, fazel@uow.edu.au

David A. Stirling

University of Wollongong, stirling@uow.edu.au

Harold C. Hill

University of Wollongong, harry@uow.edu.au

Follow this and additional works at: https://ro.uow.edu.au/infopapers

Part of the Physical Sciences and Mathematics Commons

\section{Recommended Citation}

Hesami, Amir S.; Naghdy, Fazel; Stirling, David A.; and Hill, Harold C.: Evaluation of human gait through observing body movements 2008, 341-346.

https://ro.uow.edu.au/infopapers/900

Research Online is the open access institutional repository for the University of Wollongong. For further information contact the UOW Library: research-pubs@uow.edu.au 


\title{
Evaluation of human gait through observing body movements
}

\begin{abstract}
A new modelling and classification approach for human gait evaluation is proposed. The body movements are obtained using a sensor suit recording inertial signals that are subsequently modelled on a humanoid frame with 23 degrees of freedom (DOF). Measured signals include position, velocity, acceleration, orientation, angular velocity and angular acceleration. Using the features extracted from the sensory signals, a system with induced symbolic classification models, such as decision trees or rule sets, based on a range of several concurrent features has been used to classify deviations from normal gait. It is anticipated that this approach will enable the evaluation of various behaviours including departures from the normal pattern of expected behaviour. The approach is described and the characteristics of the algorithm are presented. The results obtained so far are reported and conclusions are drawn.
\end{abstract}

\section{Keywords}

observing, body, gait, human, evaluation, movements

Disciplines

Physical Sciences and Mathematics

\section{Publication Details}

S. Hesami, F. Naghdy, D. A. Stirling \& H. C. Hill, "Evaluation of human gait through observing body movements," in International Conference on Intelligent Sensors, Sensor Networks and Information Processing, 2008, pp. 341-346. 


\title{
Evaluation of Human Gait through Observing Body Movements
}

\author{
Amir Hesami ${ }^{1}$, Fazel Naghdy ${ }^{1}$, David Stirling ${ }^{1}$, Harold Hill ${ }^{2}$ \\ ${ }^{I}$ School of Electrical, Computer and Telecommunication Engineering, University of Wollongong \\ Wollongong NSW 2522 Australia, \\ sah376@uow.edu.au,fazel_naghdy@uow.edu.au stirling@uow.edu.au, \\ ${ }^{2}$ School of Psychology, University of Wollongong \\ Wollongong NSW 2522 Australia, harry@uow.edu.au
}

\begin{abstract}
A new modelling and classification approach for human gait evaluation is proposed. The body movements are obtained using a sensor suit recording inertial signals that are subsequently modelled on a humanoid frame with 23 degrees of freedom (DOF). Measured signals include position, velocity, acceleration, orientation, angular velocity and angular acceleration. Using the features extracted from the sensory signals, a system with induced symbolic classification models, such as decision trees or rule sets, based on a range of several concurrent features has been used to classify deviations from normal gait. It is anticipated that this approach will enable the evaluation of various behaviours including departures from the normal pattern of expected behaviour. The approach is described and the characteristics of the algorithm are presented. The results obtained so far are reported and conclusions are drawn.
\end{abstract}

\section{INTRODUCTION}

Human gait has been studied scientifically for over a century. Some researchers such as Marey E.J [1] attached white tape to the limbs of a walker dressed in a black body stocking. Humans are able to derive rich and varied information from the different ways in which people walk and move. This study aims at automating this process. . Later Braune and Fischer [2] used a similar approach to study human motion but instead of attaching white tapes to the limbs of an individual, light rods were attached. Johansson [3] used MLDs (Moving Light Displays; method of using markers attached to joints or points of interests) in psychophysical experiments to show that humans can recognize gaits representing different activities such as walking, stair climbing, etc. Studies in psychology indicate that it is possible to identify an individual through non-verbal gestures and body movements and the way they walk. This is known as the Dynamic Finger Print of the individual which is formed by the individual gait and the body motion. The primary aim of this study is to verify the Dynamic Finger Print hypothesis and develop a methodology to objectively measure it. In this study, an alternative gait evaluation approach is being explored based on DFP. The approach builds on the work conducted over the last one hundred years on the study of human walking style. Cutting .et al. [4] introduced a biomechanical invariant for gait. Mounting incandescent bulbs to the joints in order to be able to study the gait, called cyclography technique, was implemented by Bernstein (1967).

Sherwood et al. [5] used four linear and nonlinear methods FIR, IIR, NARX and FTDNN to build a model with 6 outputs (reaction forces and torques) to model a knee movement. Franken and Veltink [6] identified the dynamics of the knee joint freely swinging lower leg system of paraplegic patients. Previdi and Carpanzano [7] studied feedback control of the knee - joint movement in paraplegic patients who have recovered partial functionality of muscles through Functional Electrical Simulation (FES). They used a polynomial NARX model for direct I/O controller and a standard LQ regulator has been defined as the starting point for designing a nonlinear gain scheduling controller. Lakanay [8] used the continuous wavelet transform (CWT) and Morlet wavelet to extract a diagnostic gait signature to find the abnormalities in walking. As described normal walking depends on a continual interchange between mobility and stability. Any abnormality restricting the normal free mobility of a joint or altering either the timing or intensity of muscle action creates an abnormal gait. SOM has been used to classify the extracted features. In another attempt Barton et al.[9] fed three - dimensional joint angles, moments and powers of the two lower limbs and pelvis to a Kohonen artificial neural network to quantify the deviation of patients' gait from normal. After the network has been trained, data from patients with gait problems were presented to the network which quantified the quality of gait in the form of a single curve by calculating the quantization error during the gait cycle. The idea of the existence of human gait signature has been widely accepted [10]. In almost all of the research mentioned above the human motion has been used to identify the individual, to find the abnormalities or to regenerate a movement in animation under that specific problem circumstances. Lee et al. [11] used sequential forward selection and neural networks and some extracted features from image data to classify the abnormality of gait. The achieved performance was $85 \%$. Chen et al. [12] designed a shoe - integrated system for human abnormal gait recognition. This used an inertial measurement unit consisting 
of 3D gyroscopes and accelerometers. Force resistors were employed to measure applied force from each foot. They used Principal Components Analysis (PCA) for feature selection and a Support Vector Machine (SVM) for classifying the selected features. Average success rate in classification per participant was $87 \%$ to $93 \%$. There little research on gait evaluation, and maximum performance is $93 \%$. PCA uses the covariance of the input features to identify orthogonal dimensions that best capture the variance of the original data. The resulting dimensions are linear combinations of the input features, but are not themselves necessarily interpretable as meaningful features of the input.

Gait abnormality is a common disorder in people with nervous system affections or neurological disorders, such as Multiple sclerosis, Parkinson's disease, Alzheimer's disease, and Myasthenia gravis (REF). There are many different types of gait abnormalities including:

- Scissor gait

- Antalgic gait

- Cerebellar ataxia

- Festinating gait

- Pigeon gait

- Propulsive gait

- $\quad$ Steppage gait

- Stomping gait

- Spastic gait

- Myopathic gait

- Magnetic gait

- Trendelenburg gait

Gait abnormality is defined as a physical malfunction. An abnormal gait is often due to disease or abnormality in one of many different areas of the body [13]. These can include vestibular disorders, which is responsible for maintaining balance, and damage of which can result in vertigo; central nervous system disorders such as multiple sclerosis and cerebral palsy; spinal cord abnormalities; peripheral nerve diseases; degenerative muscle diseases such as muscular dystrophy, neurodegenerative illnesses such as Parkinson's disease; skeletal abnormalities and diseases; arthritis; foot conditions such as plantar warts, bunions, ingrown toenails and pressure ulcers; and toxic reactions involving alcohol, drugs and allergies. Other common causes, many of which may be temporary, include trauma, fractures, haemophilia, immunization, tight or uncomfortable shoes, shin splints, an infection, tendonitis or torsion of the testes. Leg length and orientation at the hip (turned in or out slightly) are another cause of gait abnormality. In fact, for most individuals, both legs are rarely exactly the same, a condition that is especially pronounced if one of the legs was broken during childhood.

Symptoms of gait abnormality are abnormal walks such as a stooped, rigid posture, with the head and neck bent forward; legs flexed slightly at the hips and knees, giving the appearance of crouching; knees and thighs hitting or crossing in a scissors like movement; a stiff, foot-dragging walk caused by one sided, long-term, muscle contraction; or a distinctive duck-like walk. The data used in the study is obtained using a sensor suit Moven ${ }^{\circledR}$ developed by Xsens
[14]. The inertial signals are measured and inferred through an inverse kinematic model of the human body that includes 23 degrees of freedom (DOF) model across the human body. The signals include position, velocity, acceleration, orientation, angular velocity and angular acceleration. In this stage of study, the focus is on recognizing the characteristics of walk. A set of motion primitives is extracted from the captured data as the basis of the identification algorithm. The methodologies developed will be later applied to other human movements such as hand gestures, running and etc. The paper reports on the work carried out so far. The data obtained from various candidates is analyzed to extract candidate motion primitives. The aim is to develop an algorithm for motion analysis which will be added to an image processing technique to extract data in real application using cameras.

The paper is organized as follows. In Section 2 data acquisition is described. Section 3 covers the feature extraction and evaluation. In Section 4 experimental results using the models are presented, and finally the conclusion of the paper is presented in Section 5.

\section{DATA ACQUISITION}

Data is being acquired using an inertial movement suit, Moven ${ }^{\circledR}$, which provides data on 23 different segments of the body kinematics such as position, orientation, velocity, acceleration, angular velocity and angular acceleration as shown in Fig 1, Fig 2, and Fig 3.

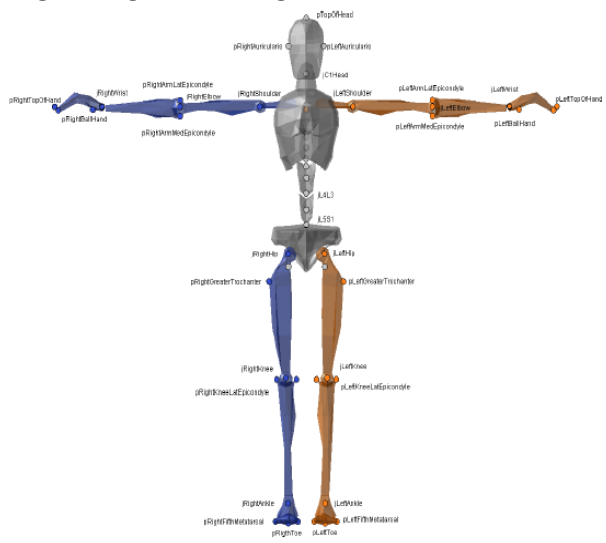

Fig. 1: Anatomical Landmarks for the Moven ${ }^{\circledR}$ suit.

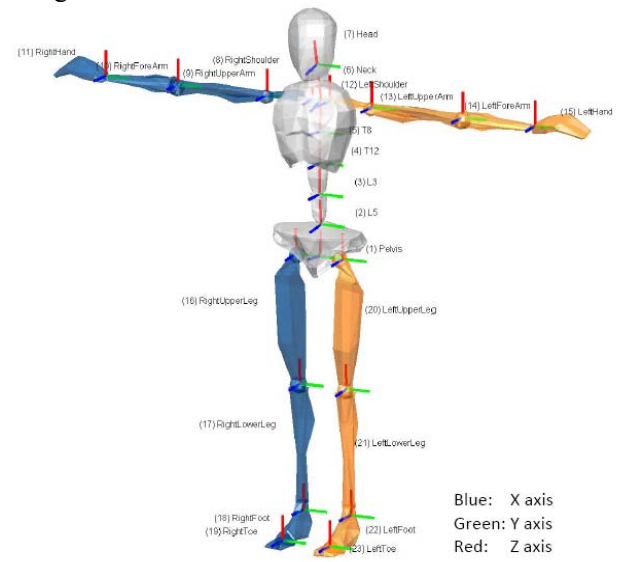

Fig. 2: Body Segments defined in the Moven $®$ suit. 




Fig. 3: Moven suit.

In capturing human body motion, no external emitters or cameras are required. As explained by [15], mechanical trackers use goniometers which are worn by the user to provide joint angle data to kinematics algorithms for determining body posture. Full 6 DOF tracking of the body segments is achieved using connected inertial sensor modules. The body segment orientation, position, velocity, acceleration, angular velocity and angular acceleration are also estimated using this method. The kinematics data is saved in an MVNX file format which is read and used, using an intermediate program coded in MATLAB.

Using the extracted features, a DFP (Dynamic Finger Print) can be generated for each individual. DFP is used to detect departure from individual's expected pattern of behaviour. Using this comparison, it is possible to find the smoothness or stiffness of the movement. In order to recognize abnormalities in gait of an individual, different measurements will be made to extract the unique Dynamic Finger Print (DFP) for that individual. The data produced by the suit consists of kinematics information associated with 23 segments of the body. The position, velocity, acceleration data for each segment will be then analyzed and a set of feature of derived will be used in classification system.

\section{FEATURE EXTRACTION}

The feature extraction section is the most important part of the research. All the classification results would be based on the extracted features. The features should be easy to extract and also must contain enough information about the dynamics of the motion. The selected features should be independent of the location, direction and path of the individual's walking. As the most important organs in walking are legs, foots, arms and elbows, the extracted features are related to these body segment's movements. Features are extracted in a gait cycle for each individual. The gait cycle is a complete stride with both legs stepping, starts with right leg as shown in Fig.4.

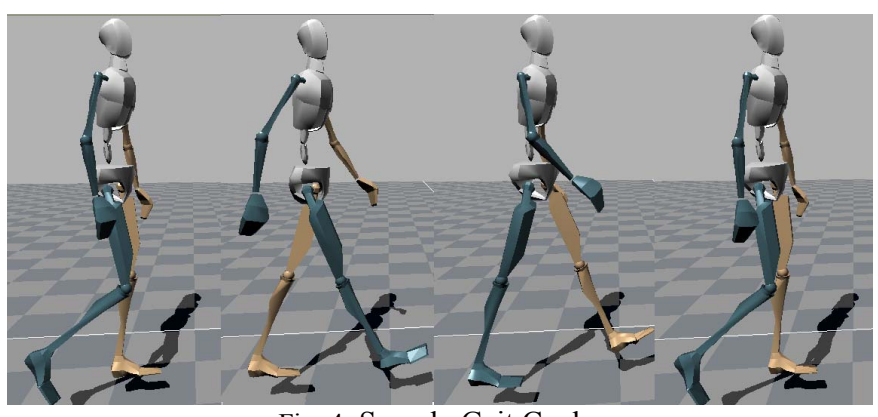

Fig. 4: Sample Gait Cycle.

The extracted features are listed below and shown as following:

- Left and Right Foot Orientation Angles.

- Left and Right Foot Angles.

- Left and Right Knee Angles.

- Left and Right Thigh Angles.

- Left and Right Elbow Angles.

- Left and Right Arm Angles.

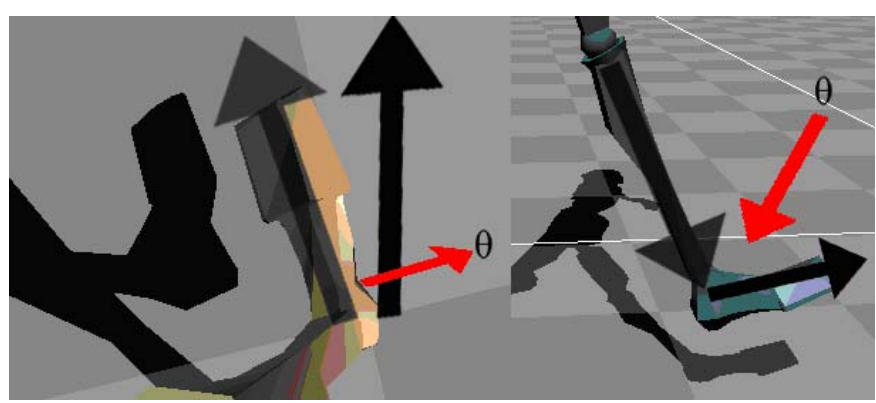

Fig. 5: Foot Orientation Angle and Foot Angle. 


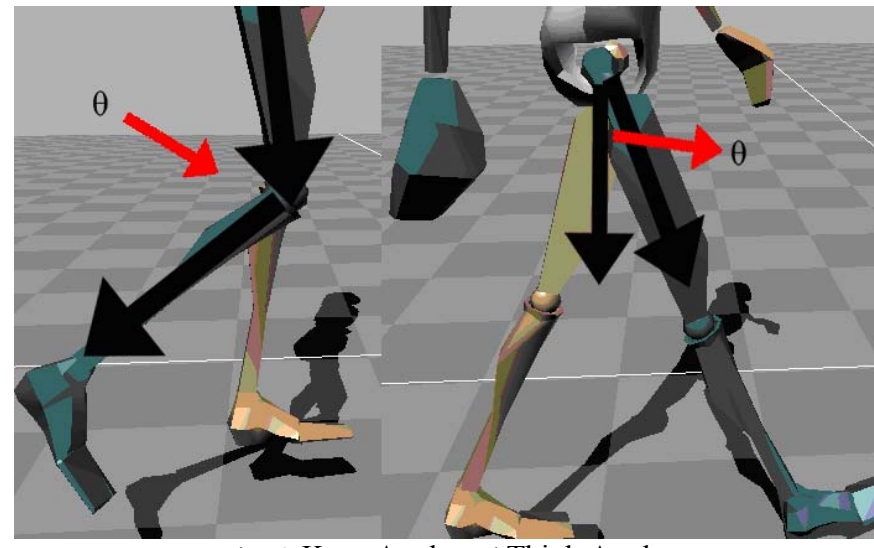

Fig. 6: Knee Angle and Thigh Angle.

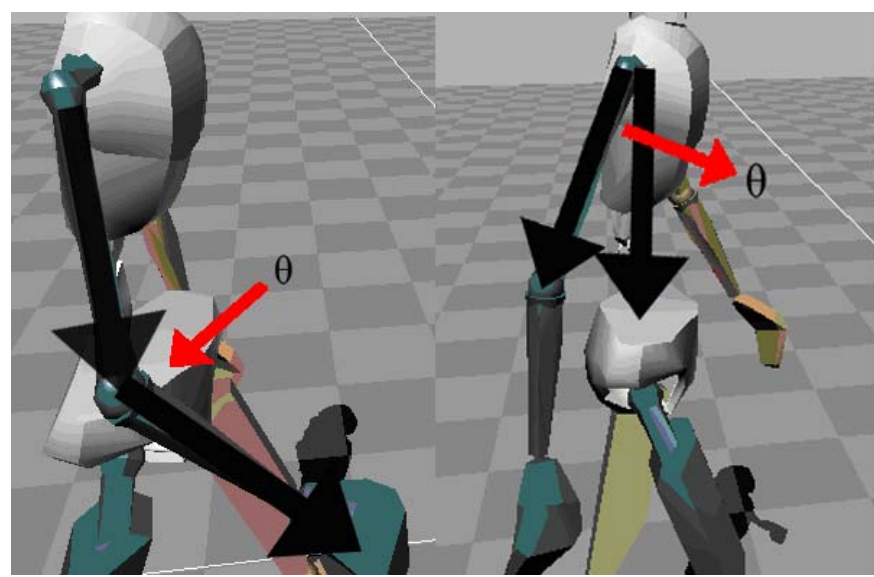

Fig. 7: Elbow Angle and Arm Angle.

Totally 12 features per individual were extracted. The extracted angles were used in radians.

\section{EXPERIMENTAL RESULTS}

The data produced by the Moven system is stored in rich detail within an MVNX (Moven Open XML format) file which contains 3D position, 3D orientation, 3D acceleration, $3 \mathrm{D}$ velocity, $3 \mathrm{D}$ angular rate and $3 \mathrm{D}$ angular acceleration of each segment in an XML format (ASCII). The orientation output is represented by quaternion formalism.

In order to examine the Dynamic Finger Print hypothesis, seven individuals wearing the Moven suit, undertook four repetitions of a simple walking task. They were asked to walk normally in one of the experiments and then walk like an old person which all of the participants limped in order to show elderly walking. A sample abnormal gait cycle is shown in Fig.8.

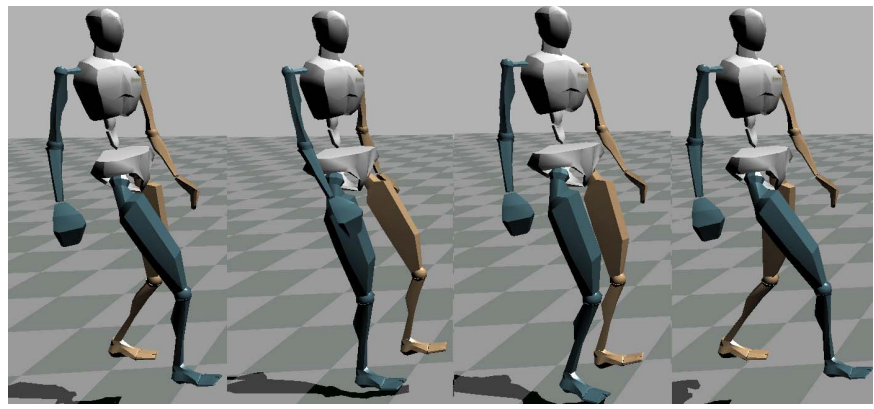

Fig. 8: Sample Abnormal Gait Cycle.

From these tasks, a range of features, across the individuals were collected and recorded as features for an identification trial. For this trial, the goal was to clearly identify an individual based on purely a combination of subtended joint angles. In addressing this recognition challenge, the rule induction system called See5 was used [16]. This system has induced symbolic classification models, such as decision trees or rule sets, based on a range of several concurrent features (attributes). The final decision trees and rule sets were created through adjustment of the various pruning options, such as setting the pruning confidence factor $(\mathrm{CF})$ to $5 \%$, and modification of the minimum number of objects to 2 . A large tree is first grown to fit the data closely and then pruned by removing parts that are predicated to have relatively high error rate. The pruning CF option affects the way that error rates are estimated and hence the severity of pruning. Values smaller than the default value which is $25 \%$ cause more of the initial tree to be pruned, while larger values result in less pruning. The minimum cases option specifies the minimum number of cases that will be maintained at the decision nodes, and essentially constrains the degree to which the induced model can fit the data. In order to get more reliable estimate of predictive accuracy $f-$ fold cross validation is used. The cases in data file are divided into f blocks of roughly the same size and class distribution. For each block in turn, a classifier is constructed from the cases in the remaining blocks and tested on the cases in the hold - out block. In this way, each case is used just once as a test case. The error rate of a classifier produced from all the cases is estimated as the ratio of the total number of errors on the hold - out cases to the total number of cases. Number of folds has been set to 35 . Once a suitable classifier performance level has been revealed using cross validation, the resultant model is generated. The options and attributes are shown in Fig. 9.

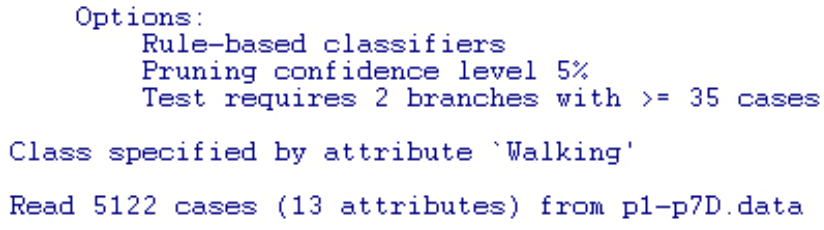

Summary of the error rates and number of rules per fold having the above attributes is shown in Fig.10: 
Evaluation on training data (5122 cases):

\begin{tabular}{rr}
\multicolumn{3}{c}{ Rules } \\
No & Errors \\
26 & $176(3.4 \%)$
\end{tabular}

\begin{tabular}{rrl} 
(a) & (b) & <-classified as \\
\hline 2365 & $--\frac{1}{88}$ & (a): class Abnormal \\
88 & 2581 & (b): class Normal
\end{tabular}

Time: 0.4 secs

Fig. 10: Classifier Summary Results.

As shown above there are two classes normal and abnormal. Participants undertaking the experiments were 3 males and 4 females between 18 to 40 years of age. According to Fig. 10, the error rate achieved is $3.4 \%$ and the number of rules is 26 . Rules are shown in Fig. 11 and Fig. 12.

\begin{tabular}{|c|c|c|c|}
\hline $\begin{array}{l}\text { Rule 1: }(317, \text { lift 2.1) } \\
\text { Left Foot } O>0.206346 \\
\text { Left Foot }>1.737376 \\
\text { Left Elbow }<=2.626953 \\
\text { Left Arm }>0.432619 \\
\quad \rightarrow \quad \text { class Abnormal } \\
\quad[0.997]\end{array}$ & $\begin{array}{l}\text { Rule } 2:(170, \text { lift } 2.1) \\
\text { Left Foot } O>0.206346 \\
\text { Right Foot }>1.874104 \\
\text { Right Knee }>2.02848 \\
\text { Right Thigh }<= \\
0.5091197 \\
\text { Right Arm }<= \\
0.1541919 \\
\text { Left Arm }>0.1742036 \\
\quad \rightarrow \text { class Abnormal } \\
\quad[0.994]\end{array}$ & $\begin{array}{l}\text { Rule } 3:(145, \text { lift 2.1) } \\
\text { Right Arm }>0.6420745 \\
\text { Left Arm }<=0.432619 \\
\rightarrow \quad \text { class Abnormal } \\
\\
{[0.993]}\end{array}$ & $\begin{array}{l}\text { Rule 4: }(225 / 1, \text { lift 2.1) } \\
\text { Left Foot } O>0.206346 \\
\text { Right Thigh }> \\
0.3693228 \\
\text { Right Thigh }<= \\
0.4899109 \\
\text { Right Arm }>0.2752825 \\
\text { Right Arm }<= \\
0.6420745 \\
\quad \rightarrow \text { class Abnormal } \\
\quad[0.991]\end{array}$ \\
\hline $\begin{array}{l}\text { Rule } 5:(90, \text { lift 2.1) } \\
\text { Right Foot } O> \\
0.06827183 \\
\text { Left Foot } O>0.206346 \\
\text { Right Arm }<= \\
0.3993336 \\
\text { Left Arm }>0.432619 \\
\quad \rightarrow \quad \text { class Abnormal } \\
\quad[0.989]\end{array}$ & $\begin{array}{l}\text { Rule } 6:(75, \text { lift 2.1) } \\
\text { Left Foot } 0<= \\
0.206346 \\
\text { Right Foot }<=2.033361 \\
\text { Left Elbow }<=2.710127 \\
\quad \rightarrow \text { class Abnormal } \\
\quad[0.987]\end{array}$ & $\begin{array}{l}\text { Rule } 7:(63, \text { lift } 2.1) \\
\text { Left Foot }<=1.531391 \\
\quad \rightarrow \quad \text { class Abnormal } \\
\\
{[0.985]}\end{array}$ & $\begin{array}{l}\text { Rule } 8:(660 / 11, \text { lift 2.1) } \\
\text { Right Foot } O> \\
0.06827183 \\
\text { Left Foot } O>0.206346 \\
\text { Right Knee }<=2.973169 \\
\text { Left Arm }>0.432619 \\
\quad \rightarrow \quad \text { class Abnormal } \\
\quad[0.982]\end{array}$ \\
\hline $\begin{array}{l}\text { Rule 9:(167/4, lift 2.0) } \\
\text { Right Foot }<=1.874104 \\
\text { Left Thigh }<= \\
0.5184123 \\
\text { Right Arm }<= \\
0.1541919 \\
\quad \rightarrow \text { class Abnormal } \\
\quad[0.970]\end{array}$ &  & $\begin{array}{l}\text { Rule 11: }(246 / 12, \text { lift } \\
\text { 2.0) } \\
\text { Right Elbow }>2.905675 \\
\text { Right Elbow }<= \\
3.027221 \\
\text { Left Elbow }<=3.013905 \\
\text { Left Arm }<=0.432619 \\
\quad \rightarrow \text { class Abnormal } \\
\quad[0.948]\end{array}$ & $\begin{array}{l}\text { Rule 12: (360i19, lift } \\
\text { 2.0) } \\
\text { Left Thigh } \varsigma= \\
0.5184123 \\
\text { Right Elbow }>3.032689 \\
\text { Right Arm } \varsigma= \\
0.1541919 \\
\quad \rightarrow \text { class Abnormal } \\
\quad[0.945]\end{array}$ \\
\hline 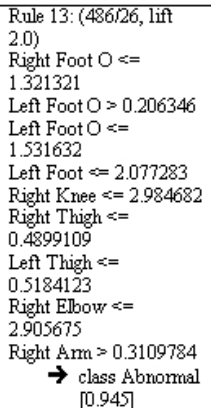 & $\begin{array}{c}\text { Rule 14: }(12019 \text {, lift } 1.9) \\
\text { Right Foot } O>1.52407 \\
\text { Right Edhow }>3.027221 \\
\rightarrow \text { class Abnomal } \\
{[0.918]}\end{array}$ & $\begin{array}{c}\text { Rule } 15:(35 / 3 \text {, lift } 1.9) \\
\text { Left Foot } O=0.206346 \\
\text { Right Elbow }>2.99114 \\
\text { Left Elbow }>3.013905 \\
\text { Right } A m>0.1541919 \\
\text { Left Arm }>0.1855841 \\
\quad \rightarrow \text { class Abnormal } \\
\quad[0.892]\end{array}$ & $\begin{array}{l}\text { Rule 16: (377155, lift } \\
\text { 1.8) } \\
\text { Right Foot } O> \\
1.321321 \\
\text { Right Foot } O<= \\
1.408431 \\
\text { Left Foot } O>0.206346 \\
\text { Right Elbow }<= \\
3.027221 \\
\text { Right Amm }>0.2752825 \\
\quad \rightarrow \text { class Abnomnal } \\
\quad[0.852]\end{array}$ \\
\hline
\end{tabular}

Fig. 11: Rules.

Each rule consists of a rule number which is used only to identify the rule, and statistics $(\mathrm{n}$, lift $\mathrm{x})$ or $(\mathrm{n} / \mathrm{m}$, lift $\mathrm{x})$ that summarize the performance of the rule. $\mathrm{N}$ is the number of training cases covered by the rule and $\mathrm{m}$, if it appears, shows how many of them do not belong to the class predicted by the rule. The rules accuracy is estimated by the Laplace ratio $(n-m+1) /(n+2)$. The lift $\mathrm{x}$ is the result of dividing the rule's estimated accuracy by the relative frequency of the predicted class in the training set. Each rule has one or more conditions that must all be satisfied if the rule is to be applicable. The class predicted by the rule is show after the conditions, and a value between 0 and 1 that indicates the confidence with which this prediction is made is shown in brackets.

\begin{tabular}{|c|c|c|c|}
\hline $\begin{array}{l}\text { Rule } 17:(342 \text {, lift } 1.9) \\
\text { Left Foot } \mathrm{O}<= \\
0.206346 \\
\text { Right Foot }>2.033361 \\
\quad \rightarrow \text { class Nomal } \\
\quad[0.997]\end{array}$ & $\begin{array}{l}\text { Rule } 18:(190, \text { lift } 1.9) \\
\text { Right Foot } O<= \\
0.06827183 \\
\text { Left Amm }>0.432619 \\
\rightarrow \text { class Normal } \\
{[0.995]}\end{array}$ & \begin{tabular}{|c|} 
Rule $19:(151$, lift 1.9$)$ \\
Right Knee $>2.973169$ \\
Left Elbow $>2.626953$ \\
Right Arm $>0.3993336$ \\
Left Arm $>0.432619$ \\
$\rightarrow$ class Normal \\
{$[0.993]$}
\end{tabular} & $\begin{array}{l}\text { Rule 20: }(49615 \text {, lift 1.9) } \\
\text { Left Foot } \mathrm{O}<= \\
0.206346 \\
\text { Left Elbow }>2.710127 \\
\rightarrow \text { class Normal } \\
\quad[0.988]\end{array}$ \\
\hline $\begin{array}{c}\text { Rule 21: }(43, \text { lift } 1.9) \\
\text { Left Foot } \approx=1.737376 \\
\text { Right Knee }>2.973169 \\
\text { Left Arm }>0.432619 \\
\rightarrow \text { class Normal } \\
{[0.978]}\end{array}$ & 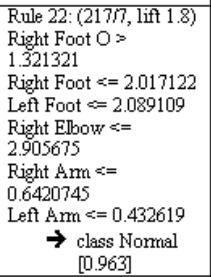 & $\begin{array}{l}\text { Rule 23: (23718, lift 1.8) } \\
\text { Right Foot O }= \\
1.408431 \\
\text { Right Ebow }<= \\
2.905675 \\
\text { Right Anm } s= \\
0.6420745 \\
\text { Left Anm }<=0.432619 \\
\quad \rightarrow \text { class Normal } \\
\quad[0.962]\end{array}$ & $\begin{array}{l}\text { Rule } 24:(345 / 16, \text { lift } \\
\text { 1.8) } \\
\text { Left Foot } O>0.206346 \\
\text { Right Elbow }<= \\
2.905675 \\
\text { Right Arm } \varepsilon= \\
0.2752825 \\
\quad \rightarrow \text { class Normal } \\
\quad[0.951]\end{array}$ \\
\hline $\begin{array}{l}\text { Rule 25: (43i5, lift 1.7) } \\
\text { Left Foot }>1531391 \\
\text { Right Thigh }> \\
0.5091197 \\
\text { Right Elow }<= \\
3.032689 \\
\text { Right Arm }<= \\
0.1541919 \\
\text { Left Amm }>0.1742036 \\
\quad \rightarrow \text { class Nomal } \\
\quad[0.867]\end{array}$ & $\begin{array}{l}\text { Rule } 26:(3826 / 1622, \text { lift } \\
\text { 1.1) } \\
\text { Left Arm } s=0.432619 \\
\quad \rightarrow \text { class Normal } \\
\quad[0.576] \\
\text { Default class: Normal }\end{array}$ & & \\
\hline
\end{tabular}

Fig. 12: Rules.

Every extracted feature has been participated in creating the rules. The importance of the features and frequency of them being used in the rules is shown in Table 1.

TABLE 1: USAGE OF FEATURES

\begin{tabular}{|c|c|c|}
\hline Feature & $\begin{array}{lr}\begin{array}{l}\text { Number } \\
\text { times }\end{array} & \text { of } \\
\text { refeing } \\
\end{array}$ & $\begin{array}{l}\text { Percentage of } \\
\text { usage/all } \\
\text { features }\end{array}$ \\
\hline Right Arm & 16 & $16 \%$ \\
\hline Left Arm & 14 & $14 \%$ \\
\hline $\begin{array}{c}\text { Left Foot } \\
\text { Orientation }\end{array}$ & 14 & $14 \%$ \\
\hline Right Elbow & 12 & $12 \%$ \\
\hline $\begin{array}{l}\text { Right Foot } \\
\text { Orientation }\end{array}$ & 10 & $10 \%$ \\
\hline Left Foot & 6 & $6 \%$ \\
\hline Right Knee & 6 & $6 \%$ \\
\hline Right Thigh & 6 & $6 \%$ \\
\hline Left Elbow & 6 & $6 \%$ \\
\hline Right Foot & 5 & $5 \%$ \\
\hline Left Thigh & 4 & $4 \%$ \\
\hline Left Knee & 1 & $1 \%$ \\
\hline
\end{tabular}




\section{CONCLUSIONS}

The primary aim of this study was to evaluate a person's gait based on a combination of angles subtended by the feet, knees, thighs, arms, and elbows. In this process 12 features were extracted, a decision tree constructed and trees converted into collection of rules called rulesets. $96.6 \%$ classifier accuracy was achieved. The participants were 3 males and 4 females between 18 to 40 . The two most important features being used by the classifier are right and left arm angles. These account for a total of $30 \%$ of the times in rules that a feature is referred to. The features could be used in patient's gait evaluation and finding the abnormalities in walking. The algorithm could also be extended to calculate departures from the normal pattern of any other expected behaviour.

\section{REFERENCES}

[1] Centre Nationale d'Art Moderne, E-J Marey 1830/1904: La Photofraphie Du Movement Paris Centre Georges Pompidou, Musee national d'art moderne, Paris.

[2] W. Braune, O. Fischer, Der Gang Des Menschen/ The Human Gait, Springer, Berlin 1904.

[3] G.Johansson, "Visual Perception of biological motion and a model for its analysis", Percept. Psycophys. 14(1973) 210-211.

[4] James E.Cutting, Dennis R.Proffitt, and Lynn T.Kozlowski, A Biomechanical Invariant for Gait Perception. Journal of Experimental Psychology: Human Perception and Performance 1978, Vol.4, No. 3, $357-372$.

[5] Jesse Sherwood, Reza Derakhshani, Trent Guess.A Comparative Study of Linear and Nonlinear Data Driven Surrogate Models of Human Joints.University of Missouri - Kansas City, 2008.

[6] Henry M. Franken, Peter H. Veltink, Member, IEEE, Roel Tijsmans, Henk Nijmeijer, Senior Member, IEEE, and Herman B. K. Boom, Associate Member, IEEE. Identification of Passive Knee Joint and Shank Dynamics in Paraplegics Using Quadriceps Stimulation. IEEE Transactions on Rehabiliation Engineering, VOL. I. NO. 3. Sep 1993.

[7] Fabio Previdi and Emanuele Carpanzano. Design of a Gain Scheduling Controller for Knee-Joint Angle Control by Using Functional Electrical Stimulation. IEEE Transactions on Control Systems Technology, VOL. 11, NO. 3, May 2003.

[8] H. Lakany, Extracting a diagnostic gait signature, Pattern Recognition, Vol.41 (2007), pp.1627 - 1637, Elsevier.

[9] Gabor Barton, Paulo Lisboa, Adrian Lees, and Steve Attfield, Gait quality assessment using self - organizing artificial neural networks, Gait \& Posture Vol.25 (2007), pp.374 - 379, Elsevier.

[10]H. Lakany, A generic kinematic pattern for human walking, Neurocomputing 35 (2000) 27 - 54.

[11] H. Lee, L. Guan, and J. A. Burne, Human Gait and posture analysis for diagnosing neurological disorders, School of Electrical and Information Engineering, Department of Biomedical Science, University of Sydney.

[12]Meng Chen, Bufu Huang, and Yangsheng Xu, Intelligent Shoes for Abnormal Gait Detection, 2008 IEEE International Conference on Robotics and Automation Pasadena, CA, USA, May 19-23, 2008.

[13] http://www.caringmedical.com/conditions/Gait_Abno rmality.htm.

[14] Xsens Technologies B.V., Moven ${ }^{\circledR}$, wireless inertial motion capture, www.xsens.com .

[15] Daniel Roetenberg, Henk Luinge, and Per Slycke. Moven: "Full 6DOF Human Motion Tracking Using Miniature Inertial Sensors", Xsen Technologies, December, 2007.

[16]RuleQuest Research: data mining tools, www.rulequest.com. 